Fabrikplanung mit CAD und Simulation

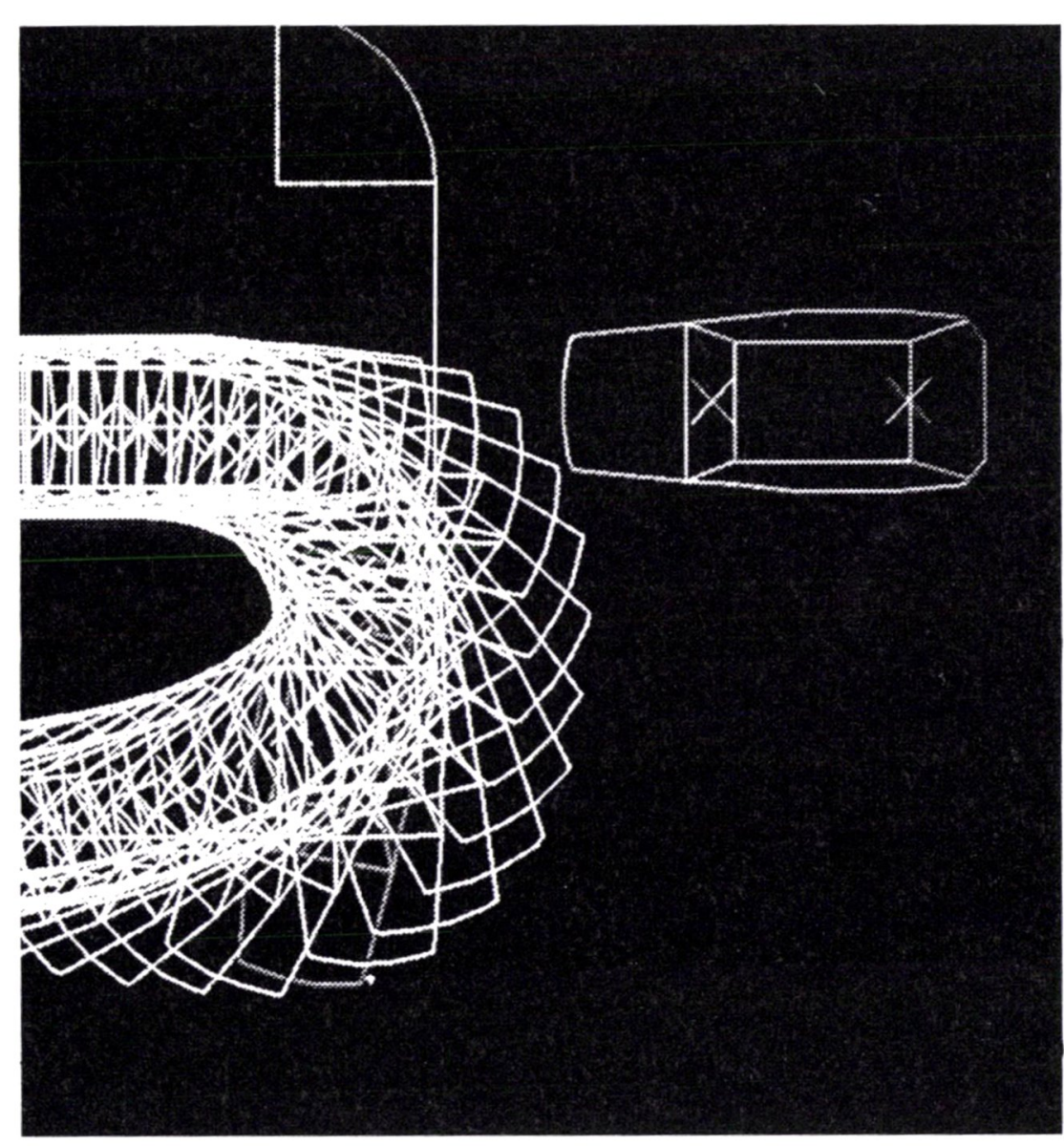

CAD und Simulation sind heute die wichtigsten Werkzeuge der Fabrikplanung. In diesem Beitrag wird die Kopplung von CAD mit der graphischen und logistischen Simulation bei der Planung von Transportsystemen dargestellt.

\section{Rechnerintegrierte Planungskette}

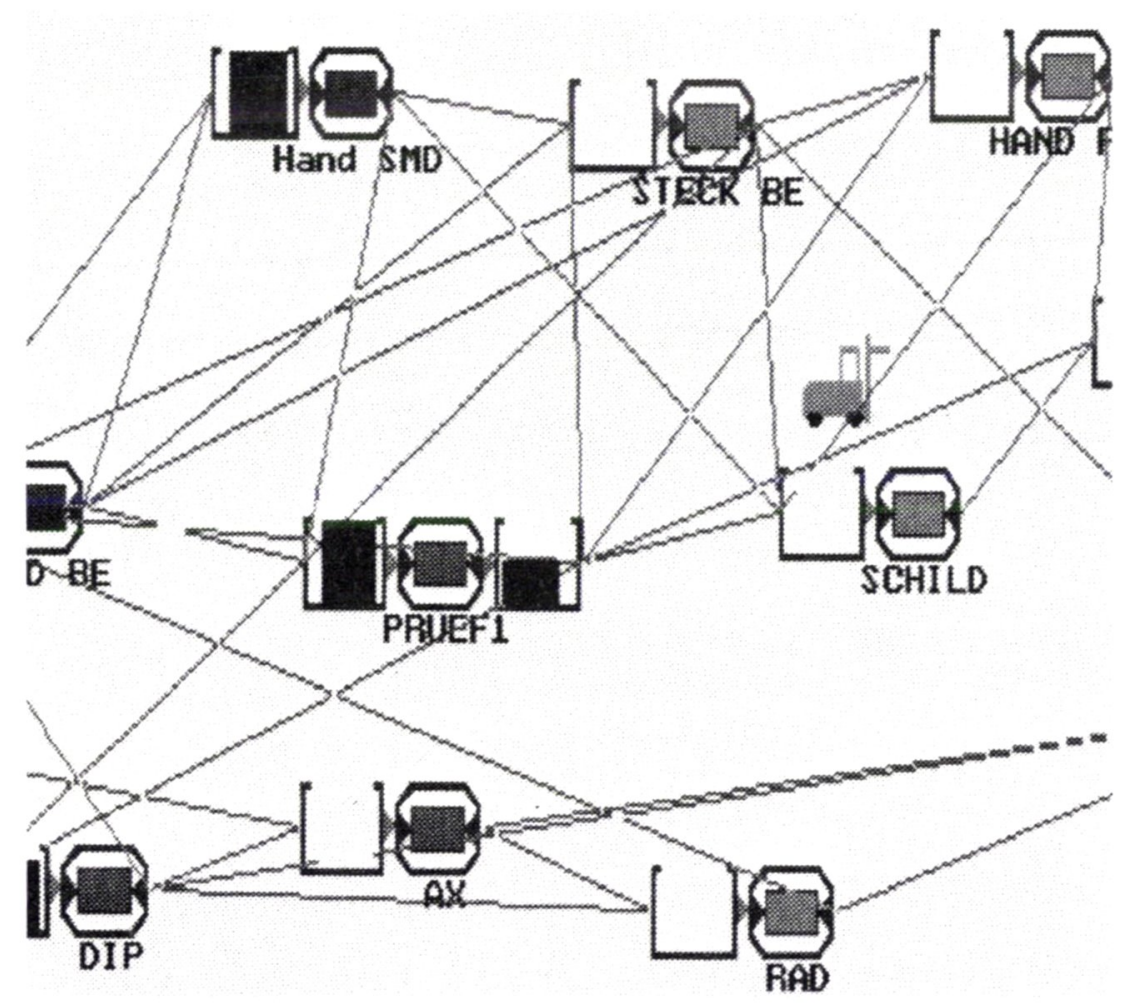

Diese Planungskette umfaßt die Datenaufbereitung, die Simulation der Fertigungsabläufe, die Layoutentwicklung und Materialflußanalyse sowie die Simulation der Transportmittel.

\section{Editorial}

Rechnerunterstützte Fabrikplanung

\section{Rechnerunterstützte Konstruktion und Planung}

T. T. Pham, M. Prestel: Vom CAD-Layout zum Simulationsmodell 296

V. Horn, J. Hein: Komplexe Produktionssysteme planen

A. Hansmann, W. Kleiner: Speditionsanlagen rechnergestützt konzipieren.

A. Jäger, M. Schöpf: Komplexe Produktionsanlagen am CADBildschirm planen.

M. Wolf: Externe Planungsfirmen in die Fabrikplanung einbeziehen ........................... 314

D. v. Zeeland: Fertigungslogistik interaktiv planen . . . . . . . 317

D. Langner: Entwicklung der rechnergestützten Fabrikplanung . . . 321

M. Frank, S. Neuthe: Fertigungssysteme modellieren und simulieren.

\section{Unternehmensplanung}

E. F. Schimke: Potentialermittlung als unternehmerische Aufgabe zur Zukunftssicherung

\section{Fabrikanlagen und Materialfluß}

M. Feige: Neuheiten der Montage- und Handhabungstechnik

\section{Kurzberichte}

Programmier-Schnittstelle erhöht die CAD-Produktivität. . . . . . . 299

Software für zentrales Maschinenmanagement . . . . . . . . . . . . 304 MAP-kompatible Kommunikation . . . . . . . . . . . . . . . . . 309

Bewegungssysteme mit PCs steuern . . . . . . . . . . . . . . . 309

Neue Drehmaschine für kleine und mittlere Losgrößen . . . . . . . . 316

Leitfaden zur Steuerung von Montagezellen . . . . . . . . . . . . . . . . . . 320

Integrierte Planung von Fabrikanlagen. . . . . . . . . . . 325

PPS und Simulationssoftware mit Expertensystem koppeln . . . . . . 325

CNC-Transfer-Bearbeitungsmaschine . . . . . . . . . . . . 329

Werkzeugspannung mit Kurzkegel und Plananlage . . . . . . 333

Integriertes System für die Komponentenfertigung . . . . . . . . . 337

\section{Buchbesprechungen}

\section{Notizen}

Veranstaltungen. . . . . . . . . . . . . . . . . . . . . . . . . . 339

Fabrikanlagen und Materialfluß . . . . . . . . . . . . . . . 340

$\begin{array}{ll}\text { Impressum } & 340\end{array}$

\title{
Graphische Layoutplanung
}

Das an einem Hochschulinstitut entwickelte Planungs-Leitsystem besteht aus einer Datenbank zur Integration der verschiedenen Planungswerkzeuge und einem Layoutplanungssystem auf Basis eines verbreiteten CAD-Systems. 


\section{Beiblatt: Laser-Praxis}

Notizen

Leitartikel

P. Wirth: Führungswechsel in der Laser-Materialbearbeitung

LS 9

\section{Laser-Metallbearbeitung im Maschinen-, Fahrzeug- und Stahlbau}

G. Eberl, U. Sutor: Laser-Abtragen - eine neue Technologie LS 11

J. Bock: Laser zum wirtschaftlichen flexiblen Blechbearbeiten

U. W. Hunziker, A. P. Schwarzenbach: Einfach arbeiten in jeder Dimension.

\section{Laser-Feinbearbeitung}

R. Erxleben: Der Laser verschließt leichte Hybrid-Gehäuse

H.-E. Kiecker, S. Luber: Laser-Anwendung in der KleinschützFertigung

G. Häusele, F. Tuma: Keramik unterm Laserstrahl

K. G. Hänsel: Innovativer Werkstoff Glas - innovativ trennen . . . LS 45

\section{Laser-Beschriften,-Markieren}

W. Numberger: Strichcodes individuell und rationell geschrieben LS 48 W. Grünwald: Lasermarkierung in der Elektronik-Industrie beliebt LS 51

\section{Laser in der industriellen Meßtechnik und Qualitätssicherung}

M. Bottlinger, A. Ortlinghaus: Signale richtig interpretieren - Fehlstellen auf metallischen Oberflächen optisch messen

H.-P. Hippler: Länge, Breite, Dicke mit dem Laser messen Systemlösungen sparen Zeit und Geld

H. Petry: Schichtdicken mit dem Laser prüfen - neue Möglichkeiten durch photometrische Materialprüfung

H. J. Langer, H. Steinbichler: Optoelektronisches Digitalisieren von dreidimensionalen Flächen.

H. Bögel: Laser führt Schweißroboter mit Pilotsensor

\section{Kurzberichte}

Laser-Materialbearbeitung in der Automobilindustrie.

LS 10

Laserscanner zum Positionieren

LS 14

Neue Kupferdampflaser

LS 14

Alternative für den Helium/Neon-Gaslaser

LS 20

Halbleiter-Arrays mit hohem Wirkungsgrad

LS 30

Quarzverzögerungsplatten

LS 42

Neue Diodenlasergepumpte Festkörperlaser

LS 50

Sehr kleiner Justier- und Experimentierlaser

LS 50

LS 50

LS 59

Laseraktivitäten verkauft

LS 66

Kompaktlaser zum Gravieren und Beschriften

LS 66

Der U.S.-Markt für Industrielase

LS 68

LS 68

LS 70

LS 70

Lasertechnik auf Rädern
LS 71
Interaktives Planungswerkzeug

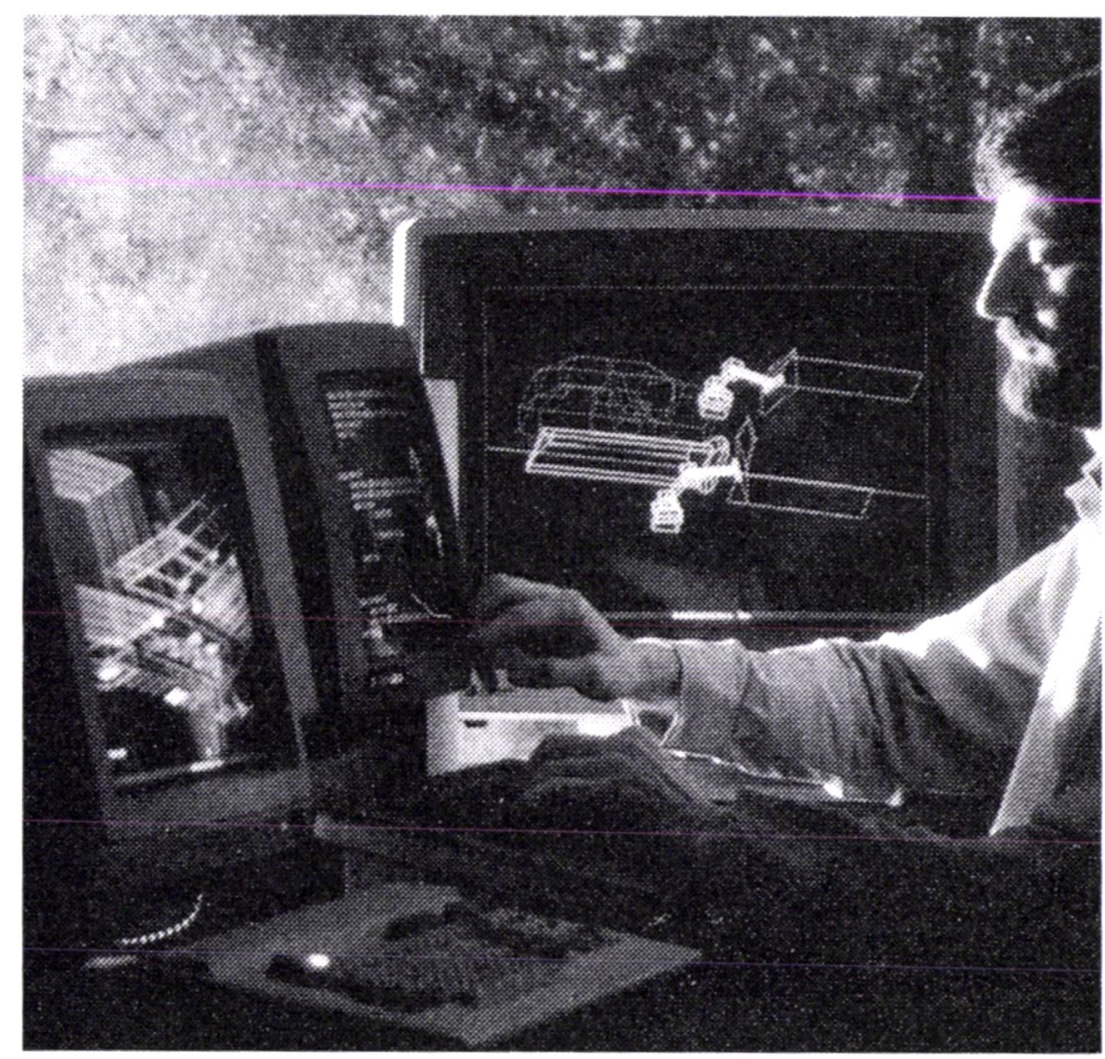

Bei dem hier beschriebenen System ist die CAD-Funktionalität mit einer prozeduralen Sprache zur Festlegung und Ablaufkontrolle des Materialflusses kombiniert. Simulationsspezifischer Programmcode kann menügesteuert erstellt werden. Gleichzeitig entsteht "automatisch" eine dreidimensionale Animation.

Fabrikplanung in der Automobilindustrie

Die Automobilindustrie ist die Bran che, in der neue logistische Material versorgungssysteme am weitesten fortgeschritten sind. Konzepte wie Just-in-Time, Materialfeindisposition oder Schnellumschlagsysteme und logistische Dienstleister erfordern entsprechende Voraussetzungen in der Werkstruktur, die nur noch mit rechnerunterstützter Fabrikplanung geschaffen werden können. S. 321

\section{Sonderteil: Beiblatt Laser-Praxis}
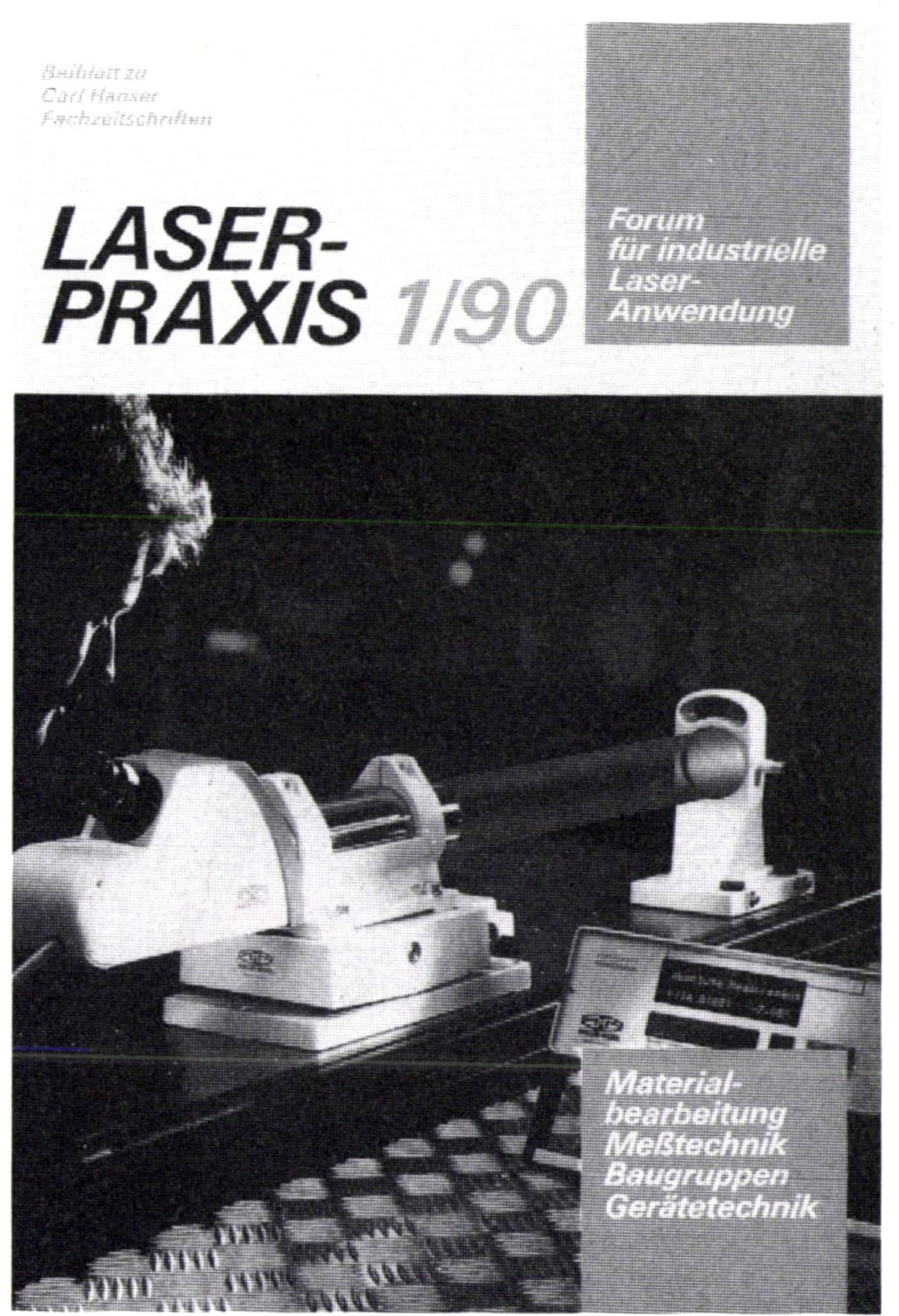